EFFECT OF GRAIN BOUNLARY STRUCTURE ON GRAIN BOUNDARY

DIFFUSIVITIES IN THE AU/AG SYSTEM

Qing Ma

R. W. Balluffi

\author{
. W. Ballutid \\ Department of Materials Science and Engineering \\ Massachusetts Institute of Technology, Cambridge, MA 02139
}

December 1, 1990
Massachusetts Institute of Technology
Cambridge, Massachuseits 02139

Prepared for

U. S. Department of Energy

under contract DE-FG02-87ER 45310

This report was prepared as an account of work sponsored ty the United States Government. Neither the United States nor the United States Dispartment of Energy, nor any of their employees, nor any of their contractors, subcontractors, or their employees, makes any warranty, express or implied, or assumes any legal liability or responsibility for the accuracy, completeness, or usefulness of any information, apparatus, product or process disciosed or represents that its use would not infringe privately owned rights. 


\section{EFFECT OF GRAIN BOUNDARY STRUCTURE ON GRAIN BOUNDARY DIFFUSIVITIES IN THE AU/AG SYSTEM}

QING MA AND R. W. BALLUFFI

Massachusetts Institute of Technology, Cambridge, MA

\section{ABSTRACT}

Grain boundary chemical diffusivities for a series of symmetric [001] tilt boundaries in the Au/Ag system were measured by the surface accumulation method using newly developed thin-film multi-crystal specimens, in which the grain boundaries feeding the accumulation surface were all of the same type. Possible effects due to segregation at the grain boundaries and surfaces were avoided. CSL boundaries of low- $\Sigma$ (i.e., $5,13,17,25$ ) and also more general boundaries with tilt angles between the low- $\Sigma$ orientations were selected. The diffusivities were found to vary monotonically with tilt angle (i.e., no cusps at low- $\Sigma$ 's were found) in a manner consistent with the Structural Unit model.

\section{INTRODUCTION}

During the last few decades, a number of studies has been made of the effect of grain boundary structure on grain boundary diffusion [1]. However, the basic characteristics of the effect for high angle boundaries remain unclear. One of the current issues is the possible existence of cusps in the grain boundary diffusion parameter, $\delta D_{b}$, versus misorientation angle, $\theta$, curve for [001] tilt boundaries at special misorientations corresponding to low- $\Sigma$ short-period boundaries. (Here, $D_{b}=$ boundary diffusivity, $\delta=$ boundary width.) Figuret displays the result of a $\mathrm{Ag}$ grain boundary self-diffusion experiment reported recently by Sommer, ef al. [2] showing a monotonic relationship between $\delta D_{b}$ and $\theta$. On the other hand, Aleshin, ef al. [3] measured the lumped chemical grain boundary diffusion parameter $s \delta D_{b}$ versus misorientation and observed deep cusps at several special misorientations (Fig. 2). This parameter includes the segregation factor, $\mathbf{s}$, which itself is also a function of boundary structure. Segregation effects, rather than variation in the $\delta D_{b}$ parameter may therefore have been responsible for the presence of the cusps in the latter case, and special efforts must be made to test this possibility.

In this report, we present measurements of chemical grain boundary diffusivities in the $\mathrm{Au} / \mathrm{Ag}$ system for a series of symmetric [001] till boundaries using a newly developed multi-crystal surface accumulation method. In this method (illustrated in Fig. 3) lattice diffusion is frozen out, and during the diffusion annealing the $\mathrm{Ag}$ atoms diffuse from the source surface to the accumulation surface through the transverse grain boundaries.

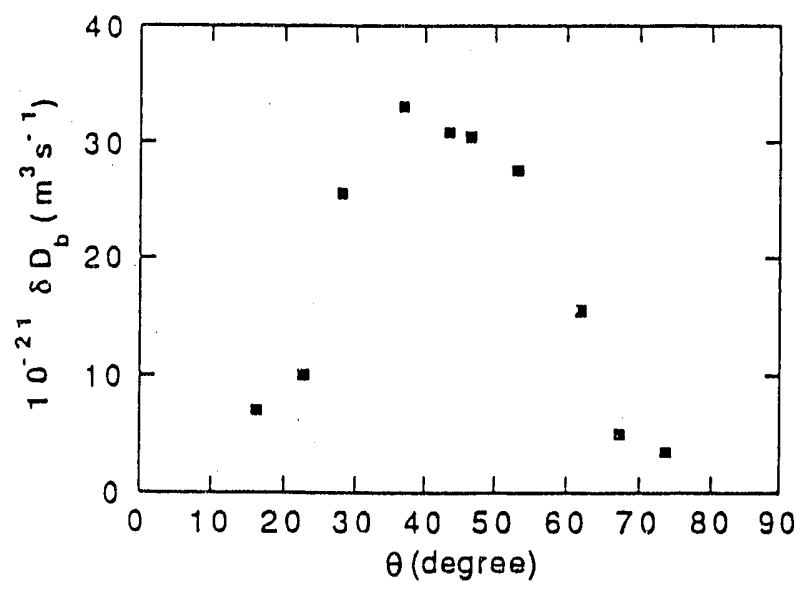

Fig. 1 Grain boundary self-diffusion of $\mathrm{Ag}$ along [001] tilt grain boundaries, after Sommer et al. [2].

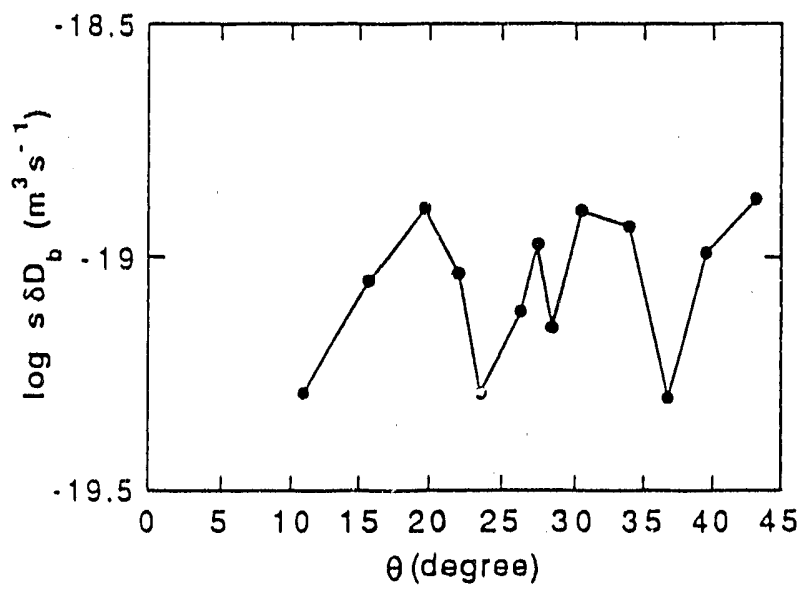

Fig. 2 Grain boundary chemical diffusion along [001] tilt grain boundaries in $\mathrm{Al} / \mathrm{Zn}$ system, after Aleshin et al. [3]. 
The chemical composition of both surfaces is monitored by means of Auger spectroscopy. The rate-limiting step is the diffusion along the boundaries, and, as shown below, this allows a direct measurement of the grain boundary diffusion parameter, $\delta D_{b}$ to be made. The technique is similar in many respects to that used earlier by Hwang and Balluffi [4] but incorporates a number of major improvements. These include a better defined constant source condition, the elimination of effects due to any segregation at the boundaries or surfaces, and the use of specially. prepared specimens in which all of the transverse boundaries are parallel to each other and are of the same type (i.e., possess the same misorientation and inclination of the boundary plane as illustrated in Fig. 4) Compared with traditional bicrystai profiling methods, this multi-crystal surface accumulation method has the following advantages: (1) ideal for studying boundary diffusion in the type-C kinetics region at relatively low temperatures; (2) capable of eliminating segregation effects; (3) allows boundary quality to be examined directly by TEM; and (4) allows repeated usage of one specimen at diffcient iemperatures.

\section{EXPERIMENTAL PROCEDURE}

The specimens were prepared by using the method illustrated schematically in Fig. 5 . The thicknesses of the $\mathrm{Au}$ and $\mathrm{Ag}$ layers were $1000 \AA$ each, and the spacing between parallel grain boundaries was $2 \mu$.

A novel rotary specimen heating stage was developed so that both specimen surfaces could be ion sputter cleaned and measured in turn for any accumulation of $\mathrm{Ag}$ or Au atoms as the result of the grain boundary diffusion.

Areas of the specimen which were judged favorable for measurement were first selected using standard TEM. After the specimen was mounted on the istary heating stage these areas could be positioned directly in front of the Auger analyzer with the help of SEM. After positioning, the sample was heated to the desired temperature and the two surfaces were successively sputter-cleaned. The gold surface was then turned perpendicular to the electron beam and the rate of $\mathrm{Ag}$ accumulation was measured. After sufficient data were obtained, the specimen was sputter-cleaned for another run. The Auger signals were calibrated by the usual relative intensity analysis of the $356 \mathrm{eV} \mathrm{Ag}$ peak, and the $69 \mathrm{eV}$ and $2024 \mathrm{eV}$ Au peaks [4].

\section{RESUL.TS}

During the measurements, it was clearly observed that the $\mathrm{Ag}$ accumulation rate was virtually constant at the early stage of diffusion as seen in Fig. 6 . This result indicates that:

(1) The system reaches a constant quasi-steady state quickly, and any significant transient is not observed.

(2) The effective surface concentration of $\mathrm{Ag}$ near the boundary exit regions is small enough in the early stage of the diffusion to preserve the constant diffusion rate. This implies that the $\mathrm{Ag}$ atoms spread out on the surface fairly uniformly and do not pile up near the exit regions. This is consistent with the fact that surface diffusion is generally much faster than grain boundary diffusion.

(3) The source surface remains pure in $\mathrm{Ag}$ to a large extent, so that it acts as an effectively constant source. This is confirmed by the observation that the Au signal from the 

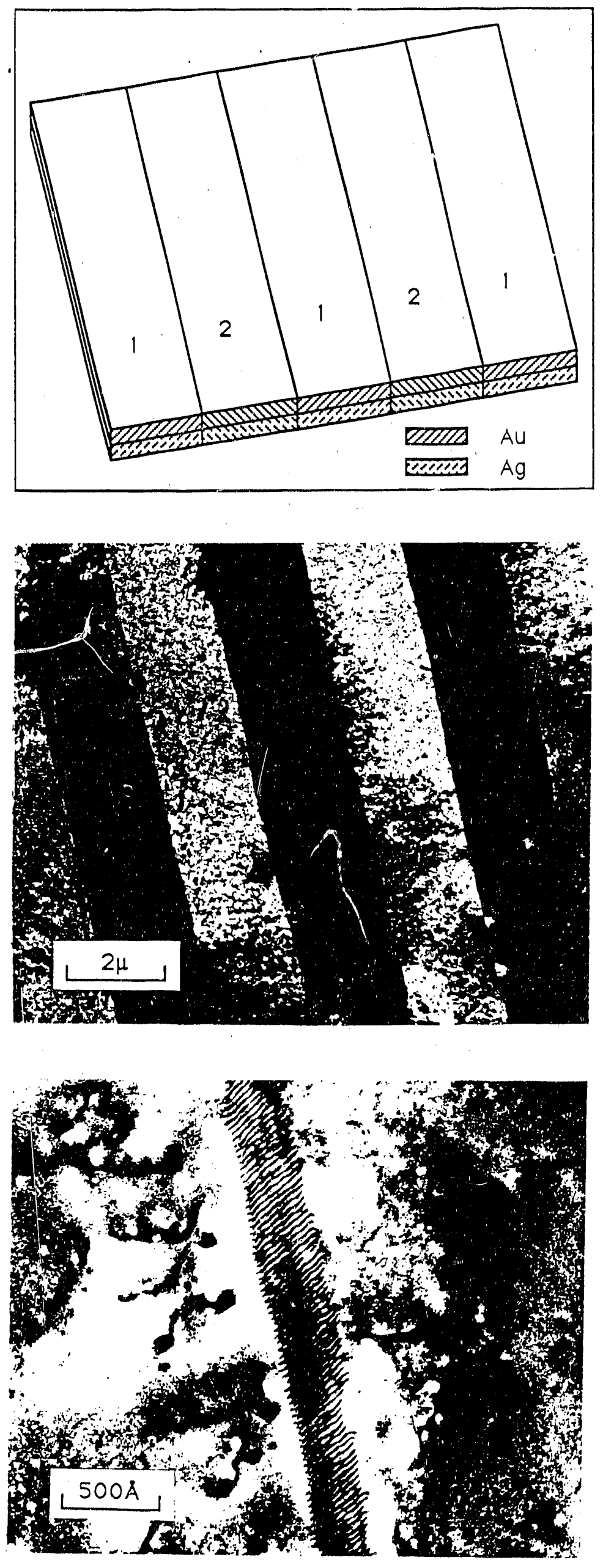

Fig. 4 Multi-crystal diffusion specimen.

(a) Schematic diagram of ideal specimen. Crystals have two orientations, i.e., 1 and 2.

(b) Top view of a typical area of specimen illustrated in (a). (c) Grain boundary edge dislocations in a [001] tilt boundary. Dislocations run parallel to tilt axis. Boundary observed obliquely. 

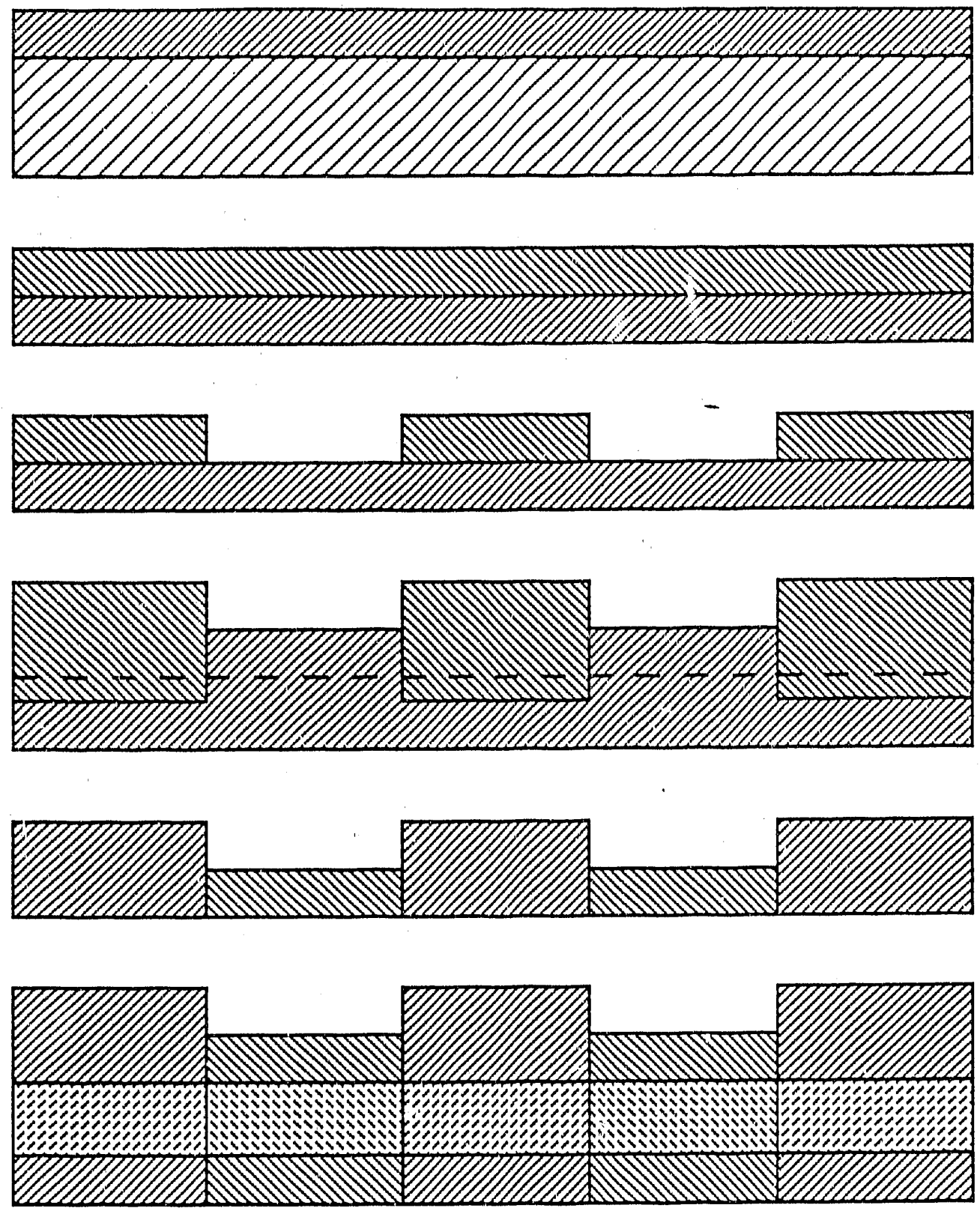

Fig. 5 Schematic diagram of diffusion specimen preparation method. (a) A (001) single crystal Au film is epitaxially grown on a $\mathrm{NaCl}$ substrate; (b) A bicrystal is then prepared by welding two thin (001) single crystal Au films together face-to-face at the desired misorientation; (c) Then, by use of high resolution lithographic techniques, an array of parallel troughs is produced; (d) Next, a layer of Au is epitaxially deposited; (e) The specimen is then ion-milled from the other side in order to remove all the material up to the dashed line in (d); ( $f$ ) Next, successive layers of $\mathrm{Ag}$ and $\mathrm{Au}$ are epitaxially deposited on the newly ion-milled surface; (g) Finally, the Au layer deposited last is isolated by dissolving the thick $\mathrm{Ag}$ layer away in nitric acid, and a thin layer of $\mathrm{Ag}$ of controlled thickness is epitaxially deposited on it to produce the final diffusion specimen. 


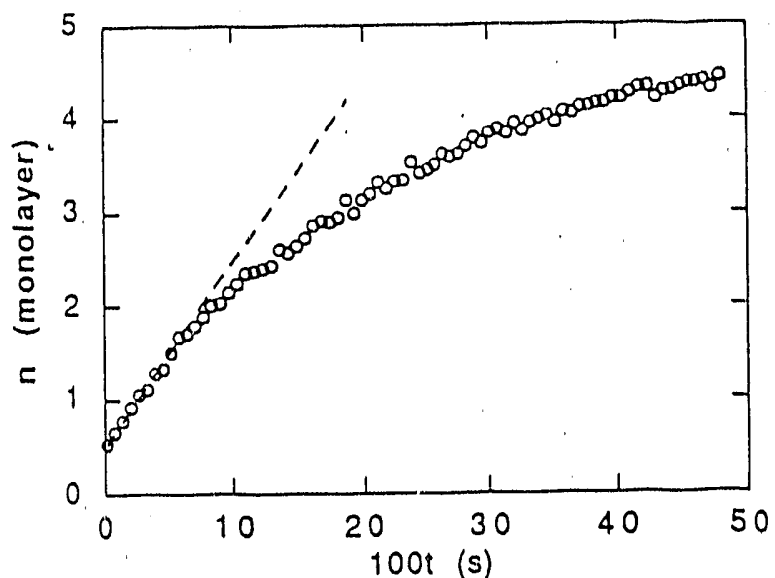

Fig. 6 Surface accumulation rate for a $\Sigma=5$ boundary at $190^{\circ} \mathrm{C}$. The dashed line represents the constant accumulation rate at the early stage of diffusion.

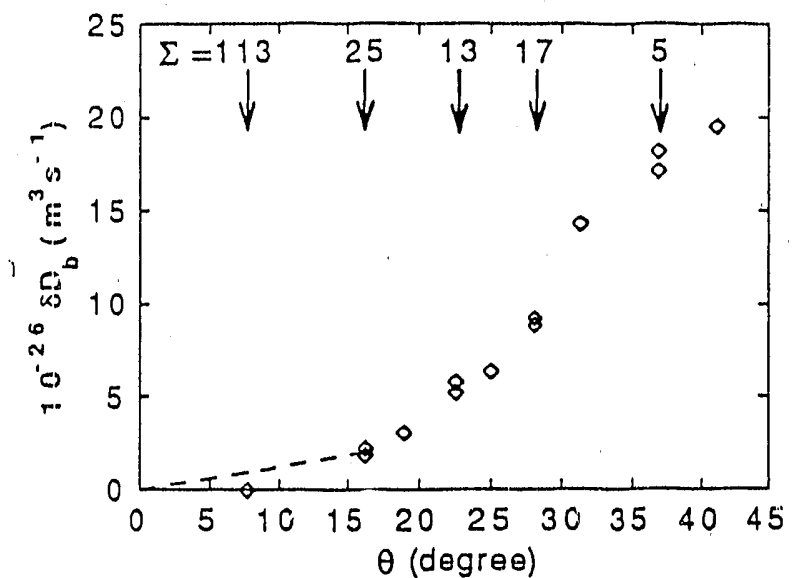

Fig. 7 Measured grain boundary diffusion parameter $\delta D_{b}$ as a function of misorientation angle $\theta$ for [001] tilt boundaries in $\mathrm{Au} / \mathrm{Ag}$ system at $190^{\circ} \mathrm{C}$.

source surface (which was measured intermittently) remained at the background noise level during the period that the $\mathrm{Ag}$ accumulated linearly on the accumulation surface.

With the above knowledge, a computer model was established to simulate the process of $\mathrm{Ag}$ atoms diffusing from the source surface through the grain boundaries to the accumulation surface. According to the model, the accumulation rate during the early diffusion stage (small surface concentration) is constant and essentially independent of the boundary to surface segregation ratio $k=C_{b} / C_{s}\left(C_{b}=\right.$ boundary concentration; $C_{s}=$ surface concentration), and is insensitive to the choice of surface layer thickness. Clearly then, the boundary diffusion parameters can be determined with little effect from segregation if the initial constant rate of accumulation is used in the calculation.

It is also confirmed that the diffusion system reaches a quasi-steady state very quickly, and that the process afterwards can be described by the following simple relations:

$$
d Q / o^{\prime} t=\delta D_{b}\left[C_{b}(0)-C_{b}(1)\right] / / b
$$

where $Q$ is the surface density of accumulated $\mathrm{Ag}, \mathrm{I}$ is the thickness of the diffusion specimen and $b$ is the spacing between adjacent boundaries.

At the beginning, $C_{b}(0)=k C_{S} \cong C_{S}$ and $C_{b}(I)=k C_{A}$, where $C_{S}$ is the concentration of the pure $A g$ source and $C_{A}$ is the $A g$ concentration on the accumulation surface. But $C_{A}$ is very small comparing to the saturation value, so that $C_{b}(I) \equiv 0$. Therefore, the initial slope of the accumulation profile is

$$
d Q / d t=\delta D_{b} C_{s} / l b
$$

and

$$
\delta D_{b}=a l b(d n / d t)
$$

where $\mathbf{a}$ is the thickness of a monolayer and $\mathbf{n}$ is the accumulated $\mathrm{Ag}$ in monolayers.

Values of $\delta \mathrm{D}_{\mathrm{b}}$ at $190^{\circ} \mathrm{C}$ were therefore determined from Eq. (3) using the measured rate of accumulation, $\mathbf{d n} / \mathbf{d t}$, and are plotted versus $\theta$ in Fig. 7. The magnitudes at the higher angles, scaled according to $T_{m} / T$, agree very well with the values previously obtained for general boundaries in the Au/Ag system in [4] and in other systems as compiled by Kaur and Gust [1].

\section{DISCUSSION}

The results in Fig. 7 indicates that $\delta D_{b}$ varies monotonically with $\theta$. No cusps at the low- $\Sigma$ short-period boundaries are evident. The general form of the $\delta D_{b}$ versus $\theta$ curve may 
be seen to be in substantial agreement with that obtained by Sommer et al. (Fig. 1). The dependence of $\delta D_{b}$ on $\theta$ for $\theta>16^{\circ}$ in both cases may be generally understood in terms of the Structural Unit model [5]. According to this model, both the structure and the diffusivity for a continuous series of boundaries are expected to vary linearly in successive ranges of misorientalion bounded by short-period boundaries.

At low angles $\left(\theta<16^{\circ}\right) \delta D_{b}$ should increase linearly with increasing $\theta$ as indicated by the dashed line in Fig. 7 according to both the Structural Unit Model and the dislocation pipe model of Turnbull and Hoffman [6] (which is essentially equivalent). However, if the boundary dislocations become widely dissociated at very small angles, as found by Darby and Balluffi [7], this linearity will break down. This seems to have been the case in Fig. 7, where $\delta D_{b}$ at $\theta=7.6^{\circ}$ was found to be exceedingly small.

Our results, in conjunction with those of Sommer,et al. [2], suggest that the cusps found in the chemical grain boundary diffusion data in Fig. 2 may have been associated with boundary segregation. The diffusion parameter plotted in Fig. 2 is the lumped parameter $\mathbf{s} \delta \mathrm{D}_{\mathrm{b}}$ which includes the segregation factor, $\mathbf{s}$, while the data in Fig. 1 and 7 represent only the parameter $\delta D_{b}$. Since $s$ is known to depend significantly on structure and has often been found to be relatively small at short period boundaries [8], it seems possible that boundary segregation may be responsible for the observed behavior. course.

Further measurements are in progress and will be reported on in more detail in due

\section{CONCLUSION}

The present work has shown that the newly developed multi-crystal surface accumulation method is capable of measuring grain boundary diffusivities with a high degree of consistency. This method made it possible, for the first time, to study structural effects on chemical grain boundary diffusion in the type-C kinetics region, and to eliminate effects due to grain boundary and surface segregation. Grain boundary diffusivities determined for a series of boundaries containing low- $\Sigma$ boundaries as well as more general boundaries show a monotonic relationship between $\delta \mathrm{D}_{\mathrm{b}}$ and tilt angle and are consistent with the Structural Unit model. It is suggested that previous observations of deep cusps in $\mathbf{s} \delta \mathbf{D}_{b}$ curves at low- $\Sigma$ misorientations for chemical diffusion may have been due to segregation effects.

\section{ACKNOWLEDGEMENT}

This work was sponsored by the Department of Energy under Contract No. DE-FG0287ER45310. Special thanks go to the CMSE Surface Analysis Central Facility and the Submicron Structures Laboratory at MIT for their generous help.

\section{REFERENCES}

1. I. Kaur and W. Gust, Eundamentals of Grain and Interphase Boundary Diffusion, 2nd ed. (Ziegler Press, Stuttgart, 1989), P275.

2. J. Sommer, Chr. Herzig, S. Mayer and W. Gust, Defect and Diffusion Forum 66-69, 843, (1989).

3. A.N. Aleshin, B.S. Bokshtein and L.S. Shvindlerman, Sov. Phys. Solid State, 19, 2051, (1977).

4. J.C.M. Hwang and R.W. Balluffi, J. Appl. Phys. 50, 1339, (1979).

5. R.W. Salluffi and A. Brokman, Scripa Met., 17, 1027, (1983).

6. D. Turnbull and R.E. Hoffman, Acta Met., 2, 419, (1954).

7. T.P. Darby and R.W. Balluffi, Phil. Mag. 36, 53, (1977).

8. R.W. Balluffi, "Grain Boundary Structure and Segregation", in Interfacial Segregation, edited by W.C. Johnson and J.M. Blakely ( ASM, Metals Park, 1979). 

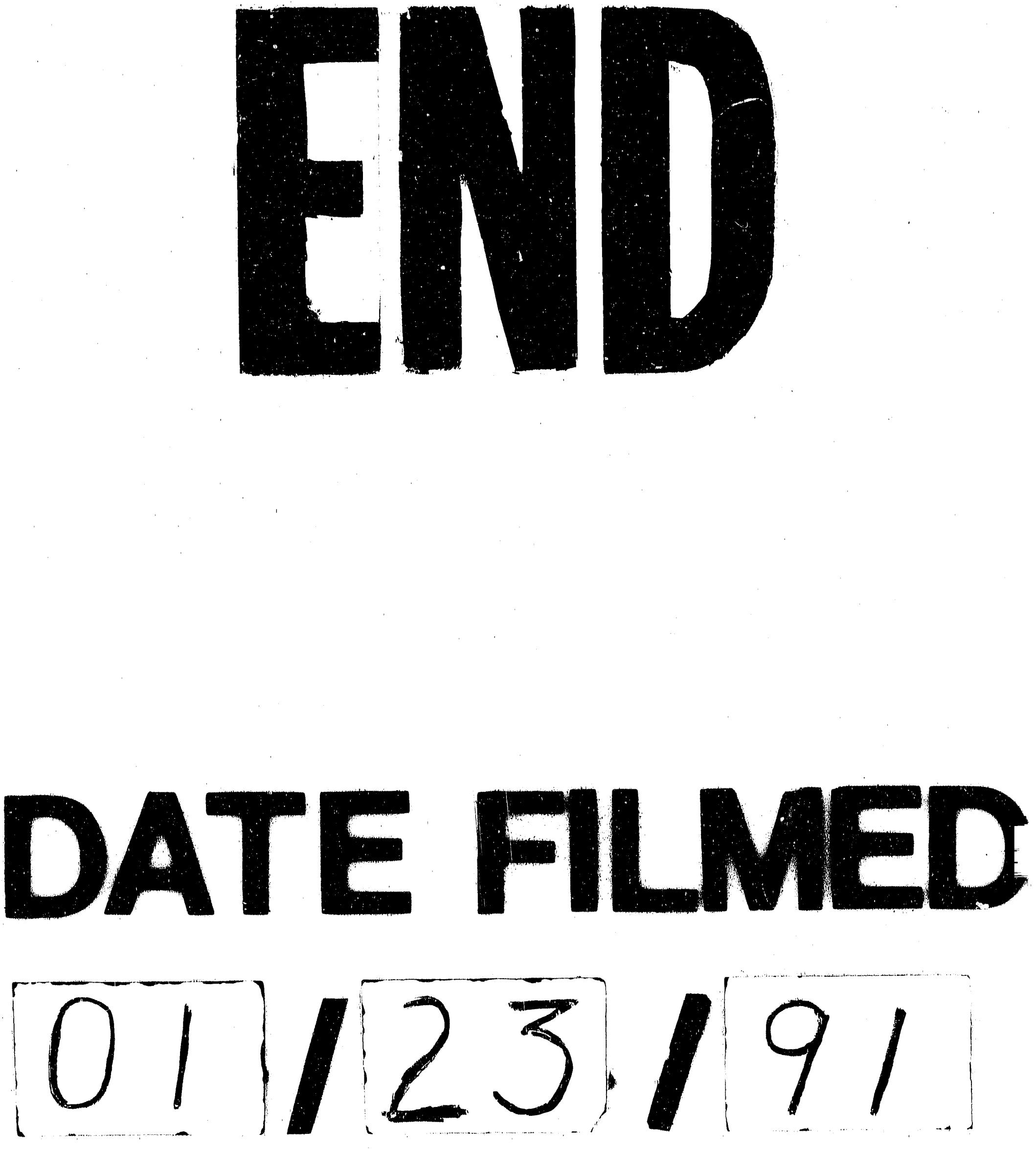
